\title{
Anisoplanatism effect on the E-ELT SCAO point spread function. A preserved coherent core across the field
}

\author{
Y. Clénet, E. Gendron, D. Gratadour, G. Rousset, and F. Vidal \\ LESIA, Observatoire de Paris, CNRS, UPMC, Université Paris-Diderot, 5 place Jules Janssen, 92195 Meudon, France
}

e-mail: yann.clenet@obspm.fr

Received 5 December 2014 / Accepted 13 September 2015

\begin{abstract}
Context. The science case studies and the optimized designing of the future adaptive optics-fed extremely large telescope instruments require the precise simulation of their adaptive optics system, potentially over their whole field of view, whatever the adaptive optics flavor the instruments will be equipped with.

Aims. We simulate the anisoplanatism effect on the extremely large telescope single conjugate adaptive optics point spread function. Our interest in this expected degradation of the correction performance with respect to the off-axis distance is in terms of the point spread function Strehl ratio and profile.

Methods. Adaptive optics simulations at the scale of extremely large telescopes are challenging given the large parameter space to explore for the adaptive optics dimensioning and the large number of degrees of freedom at play for a given set of simulation parameters. To address this problem, we have used three different simulation tools with increasing degree of fidelity compared to a real adaptive optics system. The first is based on analytical formulae and allowed us to derive the Strehl ratio degradation with the off-axis distance. The second is a Fourier-based code and provided us with both the Strehl ratio and the point spread function profile in the field. The last is an end-to-end code based on the graphical processing unit technology and also provided us with the Strehl ratio and the point spread function profile in the field.

Results. The three tools we used demonstrated a fast execution time even at the extremely large telescope scale. Cross-checks between the different codes were performed and demonstrated the coherency of the results. In addition to the expected degradation of the adaptive optics performance with the field angle, we demonstrated that in the simulation conditions applicable to the E-ELT, the single conjugate adaptive optics point spread function remains topped with a coherent core even at off-axis distances as large as 60 arcsec and consequently very low Strehl ratios. We also studied the impact of the outer scale on the point spread function profile, demonstrating that the aforementioned Airy-like pattern is observable even when the outer scale is larger than the telescope diameter.

Conclusions. These results could push for the preservation over a large field of the optical quality of any common path optics between the telescope and the instruments in single conjugate adaptive optics mode. This could offer some astrometric capabilities to these instruments operating in this adaptive optics mode.
\end{abstract}

Key words. atmospheric effects - instrumentation: adaptive optics - methods: numerical

\section{Introduction}

The first instruments on the European extremely large telescope (E-ELT, Ramsey et al. 2014) will be fed by complex multiconjugate adaptive optics (MCAO) or laser tomographic adaptive optics (LTAO) systems. The motivation is to achieve a specified $\mathrm{AO}$ correction over a given field of view and to provide greater sky coverage than a single-conjugate adaptive optics (SCAO) correction. However, these instruments are also planning to work in SCAO mode, either for full scientific exploitation (SCAO would better perform on-axis than the other AO modes) and/or in a phased approach of the AO implementation at the E-ELT.

The study presented in this article focuses on the anisoplanatism effect on the SCAO E-ELT point spread function (PSF). Resulting from the different turbulence encountered by the light propagation from the $\mathrm{AO}$ reference source and the scientific target, anisoplanatism is known to limit the correction quality of SCAO beyond the isoplanatic patch (Fried 1982). Several studies have been published about the effect of anisoplanatism on the AO performance in terms of the PSF Strehl ratio (SR) and include the influence of different turbulence characteristics: turbulence profile, outer scale, etc. (e.g. Racine \& Ellerbroek 1995; Ellerbroek 1997; Whiteley et al. 1998). Only a few studies focused on the anisoplanatism effect on the PSF profile, or the profile of its Fourier transform, the optical transfer function (Chassat 1992; Troxel et al. 1994; Fusco et al. 2000).

These studies were limited either to telescopes of diameter up to 10 meters or to small off-axis distances. More recently, studies on AO performance in the field in the ELT context are associated with wide field $\mathrm{AO}$ modes (ground layer $\mathrm{AO}$, multiconjugate AO or multi-object AO; see e.g. Neichel et al. 2008) and with the focal anisoplanatism effects on the laser guide star wavefront sensing rather than with angular anisoplanatism (e.g., Muller et al. 2011; Correia et al. 2013).

Conan et al. (2003) has shown that for large telescope diameters (larger than $\sim 30-40 \mathrm{~m}$ ) and small outer scale values (smaller than $10 \mathrm{~m}$, i.e. rather extreme cases), the turbulent PSF full width at half maximum (FWHM) approaches and then reaches the diffraction limit; this is a consequence of the reduced low order aberrations in the turbulence when the outer scale is smaller than the telescope's diameter. 
Hence, performance of SCAO in the context of the ELT has been considered only on-axis untill now. However, for the purpose of preparing the E-ELT instrument science cases, we think it is of interest to estimate the performance of SCAO across the field of view with realistic outer scale values. An additional interest is specifying the optical quality of the common path optics, if any are specific to SCAO: these optics could be optimized with respect to the SCAO performance in the field.

Our goal is to study the dependency of the AO correction with respect to the field angle for SCAO-assisted E-ELT instruments with realistic outer scale values. In order to be performed in a reasonable execution time, reliable simulations of the anisoplanatism effect have to overcome the large number of degrees of freedom of the problem (pupil sampling, number of actuators, wavefront sensor number of pixels) and - more generally for dimensioning the AO system - the large parameter space to explore (seeing variations, number of turbulent layers, AO configurations). To investigate the parameter space and validate our results on the anisoplanatism effect on the E-ELT SCAO PSF, we performed and compared three types of numerical simulations. Section 2 presents the results obtained using an analytical method derived from Chassat (1992). Section 3 presents the results obtained with a Fourier approach derived from Gendron et al. (2014) to compute PSFs and error budgets. Section 4 presents the results of end-to-end simulations using the code based on the graphical processing unit (GPU) technology and developed by Gratadour et al. (2013). We conclude in Sect. 5.

\section{Analytical estimation of the anisoplanatism effect on the E-ELT SCAO PSF}

\subsection{Computation of the analytical expression}

In order to derive a first estimation of the anisoplanatism effect on an E-ELT SCAO PSF, we have used the analytical developments made by Chassat (1992) of the anisoplanatism error expression for an $\mathrm{AO}$-corrected wavefront.

Considering an $\mathrm{AO}$ system at a telescope of radius $R$, measuring and correcting the phase up to the Zernike number $J$, observing in the direction of a scientific target $\mathrm{T}$, and measuring the aberrations in the direction of a reference source $\mathrm{R}$, Chassat (1992) estimated the residual wavefront error after AO correction in terms of the Zernike expansion coefficients $a_{j}^{\mathrm{T}}$ and $a_{j}^{\mathrm{R}}$ of the incident wavefronts received on the pupil respectively from the direction of the target and the reference source. The residual error after correction $\Delta_{C}^{2}$ is then expressed using the covariances $C_{j j}$ of these coefficients $a_{j}^{\mathrm{T}}$ and $a_{j}^{\mathrm{R}}$ (which, given the assumption of the stationarity of the turbulence, is reduced to the mathematical expectation of their product with respect to the turbulence statistics)

$$
\begin{aligned}
\Delta_{\mathrm{c}}^{2} & =\Delta_{J}^{2}+\Delta_{\text {iso }}^{2} \\
& =\sum_{j=J_{\max }+1}^{\infty} C_{j j}(0)+2 \sum_{j=2}^{J_{\max }}\left(C_{j j}(0)-C_{j j}(\alpha)\right),
\end{aligned}
$$

where $\Delta_{J}^{2}$ is the fitting error due to the finite number of degrees of freedom of the AO system, $\Delta_{\text {iso }}^{2}$ the error due to anisoplanatism, $\alpha$ the angle between the directions of the target $\mathrm{T}$, and the reference source $\mathrm{R}$ and $J_{\max }$ the highest Zernike number $j$ with the same considered radial degree; for the present article, we have used:

$\Delta_{\text {iso }}^{2}=2 \sum_{j=2}^{J_{\max }}\left(C_{j j}(0)-C_{j j}(\alpha)\right)$

Under the assumptions of the near field approximation, of statistically independent turbulence layers, of the thin screen approximation, and of Kolmogorov turbulence statistics, Chassat (1992) computed the expression of $C_{j j}(\alpha)$ :

$C_{j j}(\alpha)=\left(\frac{D}{r_{0}}\right)^{5 / 3} \frac{\int_{0}^{L} \mathrm{~d} h C_{\mathrm{N}}^{2}(h) \sigma_{j j}\left(2 \alpha h / D, 1, \pi D / L_{0}\right)}{\int_{0}^{L} \mathrm{~d} h C_{\mathrm{N}}^{2}(h)}$,

where $r_{0}$ and $L_{0}$ are the Fried and the outer scale parameters, $C_{\mathrm{N}}^{2}(h)$ the structure function of the refractive index fluctuations at altitude $h, L$ the thickness of the turbulent atmosphere, and $\sigma_{j j}\left(2 \alpha h / D, 1, \pi D / L_{0}\right)$ a function whose detailed expression is given in Appendix A.

For a discretized representation of the atmospheric turbulence layers, of depth $\Delta h_{i}$ and turbulence intensity $C_{\mathrm{N}}^{2}\left(h_{i}\right)$ at an altitude $h_{i}$, the previous equation becomes

$C_{j j}(\alpha)=\left(\frac{D}{r_{0}}\right)^{5 / 3} \frac{\sum_{i} \Delta h_{i} C_{\mathrm{N}}^{2}\left(h_{i}\right) \sigma_{j j}\left(2 \alpha h_{i} / D, 1, \pi D / L_{0}\right)}{\sum_{i} \Delta h_{i} C_{\mathrm{N}}^{2}\left(h_{i}\right)}$.

Rewriting $\% C_{\mathrm{N}}^{2}\left(h_{i}\right)=\frac{C_{\mathrm{N}}^{2}\left(h_{i}\right)}{\sum_{i} \Delta h_{i} C_{\mathrm{N}}^{2}\left(h_{i}\right)}, \Delta_{\text {iso }}^{2}$ is now expressed as follows:

$$
\begin{aligned}
\Delta_{\text {iso }}^{2}= & 2\left(\frac{D}{r_{0}}\right)^{5 / 3} \sum_{j=2}^{J_{\max }}\left(\sigma_{j j}\left(0,1, \pi D / L_{0}\right)\right. \\
& \left.-\sum_{i} \% C_{\mathrm{N}}^{2}\left(h_{i}\right) \sigma_{j j}\left(2 \alpha h_{i} / D, 1, \pi D / L_{0}\right)\right) .
\end{aligned}
$$

Hereafter, we further pushed the analytical expression of $\Delta_{\text {iso }}^{2}$ of Chassat (1992), taking advantage of some properties of the Zernike functions. By reversing the order of the sums over the $j$ and $i$ indices in Eq. (6), we expressed $\Delta_{\text {iso }}^{2}$ as sums of $\sigma_{j j}$ functions. In these sums, within a same radial degree $n$, for non-null azimuthal frequency $m$, the terms $H_{n+1,2 m, 0,14 / 3}\left(2 \alpha h / D, \pi D / L_{0}\right)$ in Eq. (A.1) for successive values of $j$ cancel each other because of the changing sign of the $K_{j j}^{1}$ function for successive values of $j$. For $m=0, \sigma_{j j}$ is reduced to a $H_{n+1,0,0,14 / 3}$ term. Hence, $\Delta_{\text {iso }}^{2}$ can be expressed as sums of $H_{n+1,0,0,14 / 3}$ terms

$$
\begin{aligned}
\Delta_{\text {iso }}^{2}= & 7.79\left(\frac{D}{r_{0}}\right)^{5 / 3} \sum_{n=1}^{n_{\text {max }}}(n+1)^{2}\left(H_{n+1,0,0,14 / 3}\left(0, \pi D / L_{0}\right)\right. \\
& \left.-\sum_{i} \% C N^{2}\left(h_{i}\right) H_{n+1,0,0,14 / 3}\left(2 \alpha h_{i} / D, \pi D / L_{0}\right)\right)
\end{aligned}
$$

with $n_{\max }$ the radial degree of $J_{\max }$. 
Table 1. Turbulence profile parameters.

\begin{tabular}{cccc}
\hline \hline $\begin{array}{c}\text { Altitude } \\
(\mathrm{m})\end{array}$ & $\begin{array}{c}\text { Thickness } \\
(\mathrm{m})\end{array}$ & $\begin{array}{c}\text { Wind speed } \\
(\mathrm{m} / \mathrm{s})\end{array}$ & $\begin{array}{c}C_{\mathrm{N}}^{2} \\
\left(10^{-15} \mathrm{~m}^{2 / 3}\right)\end{array}$ \\
\hline 47 & 94 & 15 & 2.67 \\
140 & 94 & 13 & 0.13295 \\
281 & 188 & 13 & 0.11333 \\
562 & 375 & 9 & 0.14856 \\
1125 & 750 & 9 & 0.06334 \\
2250 & 1500 & 15 & 0.00946 \\
4500 & 3000 & 25 & 0.00958 \\
9000 & 6000 & 40 & 0.00344 \\
18000 & 12000 & 21 & 0.0024 \\
\hline
\end{tabular}

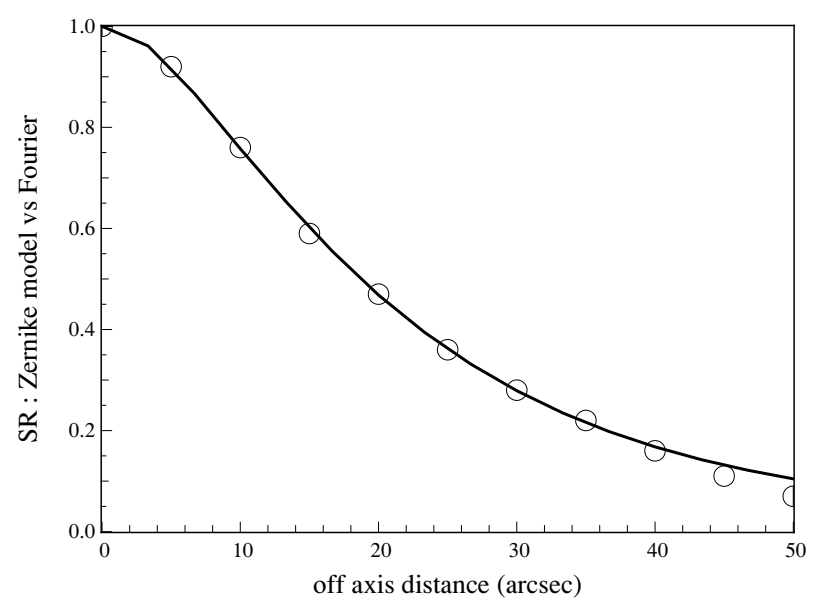

Fig. 1. $K$-band anisoplanatism Strehl ratio for an increasing off-axis distance to the reference source $\left(L_{0}=25 \mathrm{~m}\right.$, seeing $\left.=0.71^{\prime \prime}\right)$. The plain line is for the analytical estimation (Sect. 2.2) and the open circles for the pseudo-analytical estimation (Sect. 3.2).

\subsection{Application to an E-ELT SCAO PSF}

In the framework of the phase A study of the E-ELT first-light imager MICADO (Davies et al. 2010), we have estimated the anisoplanatism effect on the MICADO SCAO PSF using the analytical expression developed above. For this purpose, we have used the turbulence atmospheric parameters issued by ESO for the E-ELT instruments phase A studies (Oberti \& Kolb 2008), in the turbulence case specified by ESO for SCAO: outer scale $L_{0}$ of $25 \mathrm{~m}$, seeing at zenith and $0.5 \mu \mathrm{m}$ of $0.65^{\prime \prime}$, zenith angle of $30^{\circ}$ (i.e. effective seeing at $0.5 \mu \mathrm{m}$ of $0.71^{\prime \prime}$ and $r_{0}=14.5 \mathrm{~cm}$ ), and turbulence profile as in Table 1. We considered a telescope diameter of $D=39 \mathrm{~m}$.

Using the E-ELT M4 deformable mirror fitting error provided by ESO in Jochum (2008) for the $42 \mathrm{~m}$ telescope $\left(\right.$ sigma $_{\text {fitting }}^{2}=166 \times$ seeing $^{5 / 6}$, in $\mathrm{nm}$ rms with seeing expressed in arcseconds), we derive the maximum number of compensated Zernike modes $J_{\max }$ from the expression of the residual error resulting from the correction of these $J_{\max }$ Zernike modes by a theoretical AO system (Conan 1994): $\sigma^{2}=0.257 J_{\max }^{-5 / 6}\left(D / r_{0}\right)^{5 / 3}$ : $J_{\max }(n)=5565$ with $n=104$.

We represent in Fig. 1 the loss in Strehl ratio (normalized to the case of an on-axis correction) computed as $\exp \left(-\Delta_{\text {iso }}^{2}\right)$ against different off-axis values. We note the expected decrease in the performance with the increasing distance to the reference source: the Strehl ratio at 10 arcsec off-axis is $\sim 80 \%$ the on-axis value, while at 40 arcsec distance it reduces to $\sim 15 \%$. However, the execution speed of these analytical computations is noticeable; even at the E-ELT scale, they last less than $\sim 5 \mathrm{~s}$ for any given off-axis value on a Xeon X5690 CPU working at 3.7 GHz.

Finally, we have also computed the same curves for $J_{\max }$ ranging from radial orders $n=90$ to $n=115$ modes; the plot in Fig. 1 is hardly affected. The anisoplanatic error is mainly determined by modes with intermediate and low radial order.

\section{Pseudo-analytical estimation of the anisoplanatism effect on the E-ELT SCAO PSF}

\subsection{Computation of the pseudo-analytical expression}

A second approach has been followed to derive the anisoplanatism effect on both the Strehl ratio and the PSF profile. It is based on a Fourier code developed by Gendron et al. (2014) in a simplified version of PAOLA (Jolissaint et al. 2006) and following an original approach of Rigaut et al. (1998). The long exposure PSF is computed through the estimation of the optical transfer function (OTF), after a Fourier transform. Under the hypothesis of the stationarity of the phase, this is given by

$\operatorname{OTF}(\rho / \lambda)=\operatorname{OTF}_{\text {tel }}(\rho / \lambda) \exp \left(-\frac{1}{2} D_{\phi}(\rho)\right)$

with $\mathrm{OTF}_{\text {tel }}$ the optical transfer function of the telescope and $D_{\phi}(\rho)$ the structure function of the residual phase.

We assume that the AO error budget is made of six independent contributors:

- $\sigma_{\text {fit }}$, the deformable mirror (DM) fitting error, due to the limited number of actuators;

- $\sigma_{\text {aniso }}$, the anisoplanatism error, corresponding to $\Delta_{\text {iso }}$ defined at Eq. (3), due to the angular distance $\alpha$ between the reference source and the target source;

- $\sigma_{\text {temp }}$, the temporal error, due to the finite system bandwidth;

- $\sigma_{\text {alias }}$, the aliasing error, due to the limited wavefront spatial sampling of the wavefront sensor;

- $\sigma_{\text {noise }}$, the noise error, due to the wavefront sensor noise in its measurements;

- $\sigma_{\text {others }}$, an additional term accounting for the other contributors to the error budget that are not precisely specified at this early stage of the E-ELT project. Following Clénet et al. (2010), they are mostly the non-common path aberration error, the calibration error, the DM saturation error, the differential refraction error (different apparent locations of the reference source and the target due to the different sensing and imaging wavelengths and due to differential refraction), the chromaticism error (correction of the wavefront at a wavelength different from the imaging one), and the E-ELT segment co-phasing error.

The structure function of the residual phase is then the sum of the structure functions associated with each of the aforementioned error terms, which are computed from the power spectral density $W$ of the corresponding residual phase (Rigaut et al. 1998):

$D_{\phi}(\rho)=2 \iint W(\boldsymbol{k})(1-\cos (2 \pi \boldsymbol{k} \boldsymbol{\rho})) \mathrm{d}^{2} \boldsymbol{k}$.

To compute the different error terms detailed below, we need to define the compensation domain of the DM, noted $f_{\mathrm{DM}}$. This is 
the ensemble of spatial frequencies $\boldsymbol{k}$ that can be addressed by the DM. For a DM with a square geometry of actuators, it corresponds to frequencies with $\left|k_{x}\right|<f_{c}$ and $\left|k_{y}\right|<f_{c}$, with $f_{c}$ equal to one half of the inverse of the actuator spacing. We also note $f_{\mathrm{DM}}(\boldsymbol{k})$ the function equal to 1 when $\boldsymbol{k}$ belongs to $f_{\mathrm{DM}}, 0$ otherwise. The computation of the different error terms is explained below.

- Computation for the fitting error

$$
D_{\phi, \text { fit }}(\boldsymbol{\rho})=2 \iint_{\boldsymbol{k} \notin f_{\mathrm{DM}}} W_{\mathrm{VK}}(\boldsymbol{k})(1-\cos (2 \pi \boldsymbol{k} \boldsymbol{\rho})) \mathrm{d}^{2} \boldsymbol{k} .
$$

The integration is done over the spatial frequencies $\boldsymbol{k}$ out of the compensation domain of the DM. The spectrum $W_{\mathrm{VK}}$ is the von Kàrmàn spectrum of the turbulent phase

$$
W_{\mathrm{VK}}(\boldsymbol{k})=0.023 r_{0}^{-5 / 3}\left(k^{2}+1 / L_{0}^{2}\right)^{-11 / 6}
$$

with $k$ the modulus of $\boldsymbol{k}$.

- Computation for the anisoplanatism error

The power spectrum $W_{\text {aniso }}$ is given by

$$
W_{\text {aniso }}(\boldsymbol{k})=f_{\mathrm{DM}}(\boldsymbol{k}) \sum_{i} C_{\mathrm{N}}^{2}\left(h_{i}\right) \times\langle| T F\left(\left.\left(\phi(\boldsymbol{r})-\phi\left(\boldsymbol{r}-\boldsymbol{\alpha} h_{i}\right)\right)\right|^{2}\right\rangle,
$$

where $\phi(\boldsymbol{r})$ and $\phi\left(\boldsymbol{r}-\boldsymbol{\alpha} h_{i}\right)$ are respectively the phase of layer $i$ at the locations $\boldsymbol{r}$ and $\boldsymbol{r}-\boldsymbol{\alpha} h_{i}$ in pupil coordinates, $h_{i}$ is the altitude of the turbulent layer $i, C_{\mathrm{N}}^{2}\left(h_{i}\right)$ characterizes the strength of the layer $i$, and $\alpha$ is the angular distance vector. By developing this expression and using Fourier transform shift properties we have

$$
\begin{aligned}
W_{\text {aniso }}(\boldsymbol{k})= & f_{\mathrm{DM}}(\boldsymbol{k}) W_{\mathrm{VK}}(\boldsymbol{k}) \\
& \times \sum_{i} C_{\mathrm{N}}^{2}\left(h_{i}\right)\left|1-\exp \left(-2 i \pi \boldsymbol{k} \boldsymbol{\alpha} h_{i}\right)\right|^{2} .
\end{aligned}
$$

The phase structure function is deduced from this spectrum using Eq. (9).

- Computation for the temporal error

We assume that the temporal evolution of the wavefront in the pupil is only due to a translation (frozen flow assumption). The spatial frequencies $\boldsymbol{k}$ can then be turned into temporal frequencies $f$ using $\boldsymbol{k} . \boldsymbol{V}=f$. The residual phase power spectrum is computed by applying a factor $H_{\text {rej }}(\boldsymbol{k} . \boldsymbol{V})$ to the phase spectrum at a given spatial frequency $W(\boldsymbol{k})$ with $H_{\text {rej }}(f)$ the temporal AO rejection transfer function of the adaptive optics, a pure close loop integrator with gain in our case.

This approach assumes that the wind speed has a particular fixed direction, which usually results in a significantly elongated PSF when all wind directions of the different layers are co-aligned or when only one single layer is simulated. To the contrary, it is known and observed that, in reality, the wind direction significantly fluctuates and the boiling part is important enough so that PSFs are symmetric most of the time. Consequently, considering that the wind directions change arbitrarily, in average, the spatial frequencies are now linked to temporal frequencies by $f=k . V / \sqrt{2}$, where $k$ and $V$ are the moduli of their associated vectors. Hence, the spectrum of the residual phase for the temporal error is now obtained by applying a factor $H_{\text {rej }}(k . V / \sqrt{2})$ to the phase spectrum at a given spatial frequency $W(\boldsymbol{k})$ :

$$
W_{\text {temp }}(\boldsymbol{k})=f_{\mathrm{DM}}(\boldsymbol{k}) W_{\mathrm{VK}}(\boldsymbol{k}) \times H_{\text {rej }}(k \cdot V / \sqrt{2}) .
$$

\section{- Computation for the aliasing error}

Following Rigaut et al. (1998), we have estimated the aliasing term as approximately one-third of the fitting term. Hence, we modelled the aliasing by a flat power spectrum in the Fourier domain with an integral equal to one-third of that of fitting:

$$
\begin{array}{ccc}
\boldsymbol{k} \in f_{\mathrm{DM}} & \int_{\boldsymbol{k} \in f_{\mathrm{DM}}} W_{\mathrm{alias}}(\boldsymbol{k})=1 / 3 \int_{\boldsymbol{k} \notin f_{\mathrm{DM}}} W_{\mathrm{VK}}(\boldsymbol{k}) \\
\boldsymbol{k} \notin f_{\mathrm{DM}} & W_{\text {alias }}(k)=0 .
\end{array}
$$

\section{- Computation for the noise error}

We assume the power spectrum of noise to follow a law in $|\boldsymbol{k}|^{-2}$ with a normalizing factor to be adjusted to account for a given noise error value. Once again, this spectrum is defined over $f_{\mathrm{DM}}$ and equals 0 elsewhere.

- Computation for the "others" error

We assume here a flat power spectrum, independent of $\boldsymbol{k}$ over the domain $f_{\mathrm{DM}}$ and equal to 0 out of it, normalized so that its integral leads to $\sigma_{\text {others }}=98 \mathrm{~nm} \mathrm{rms}$, following Clénet et al. (2010).

\subsection{Comparison between the analytical and pseudo-analytical estimations}

For the purpose of the comparison between the analytical and pseudo-analytical estimations, in a first step we have considered only the anisoplanatic term of the Fourier code (Eqs. (8), (9), and (13)), and we used the simulation parameters of Sect. 2.2. We have used an actuator spacing of $0.464 \mathrm{~m}$ (i.e. $39 \mathrm{~m} / 84$ ) for the deformable mirror (leading to 5550 controlled actuators, comparable to the 5565 Zernike modes in the previous study).

The result of this comparison is shown in Fig. 1. The agreement between the two approaches is very good. It is only at large off-axis distances, which actually represent very large distance values when expressed in $\lambda / D$ unit, that the curves start to slightly deviate from each other.

We also note that these results remain consistent even when considering any actuator spacings in the range $0.4-0.5 \mathrm{~m}$; this does not have any significant impact on the anisoplanatic error term, which is dominated by the decorrelation of mediumorder modes as already mentioned. This, in turn, suggests that the compensation at large off-axis distances could largely be optimized by reducing the number of compensated modes in the command; this is, however, beyond the scope of our study.

\subsection{E-ELT SCAO PSF profiles}

In this section, we present the PSF profiles obtained with the Fourier code, now making use of all the error terms. We have considered the turbulence profile parameters in Table 1, except for the wind speed equal to $10 \mathrm{~m} \mathrm{~s}^{-1}$ for all layers. PSF profiles have been computed in the three turbulence cases of Oberti \& Kolb (2008): $r_{0}$ at zenith and $0.5 \mu \mathrm{m}$ equal to $17.2 \mathrm{~cm}(0.6 \operatorname{arcsec}$ seeing) for the "good seeing" case, to $12.9 \mathrm{~cm}$ in the "median seeing" case ( 0.8 arcsec seeing), and to $9.4 \mathrm{~cm}$ in the "bad seeing" case (1.1 arcsec seeing).

As we want to concentrate on performance degradation due to isoplanatic effects, we have only considered a single case of high signal-to-noise ratio for the wavefront sensing. The choice of our parameters result from some optimization performed during the MICADO phase A study (Clénet et al. 2010), where we had assumed a given reference source of $m_{\mathrm{V}}=12$ and an average throughput for the optical train. This reference source 
Y. Clénet et al.: Anisoplanatism effect on the E-ELT SCAO point spread function. A preserved coherent core across the field
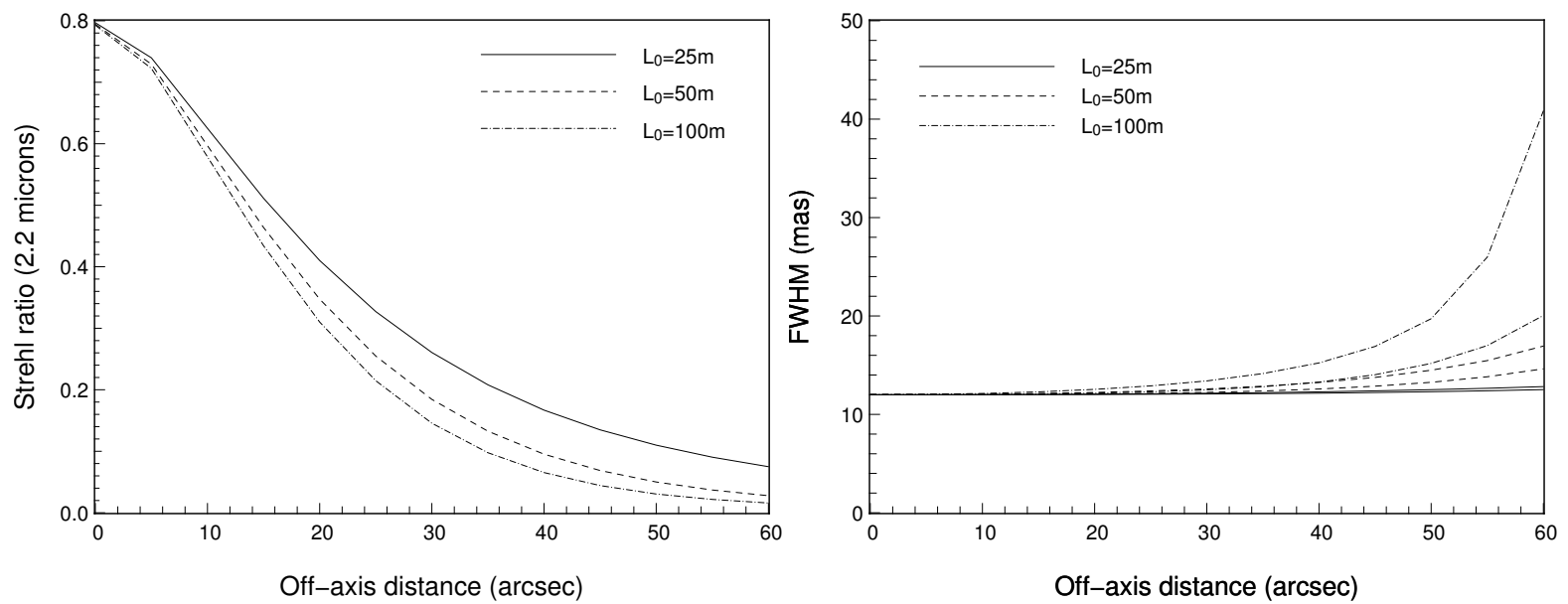

(a) Good seeing case
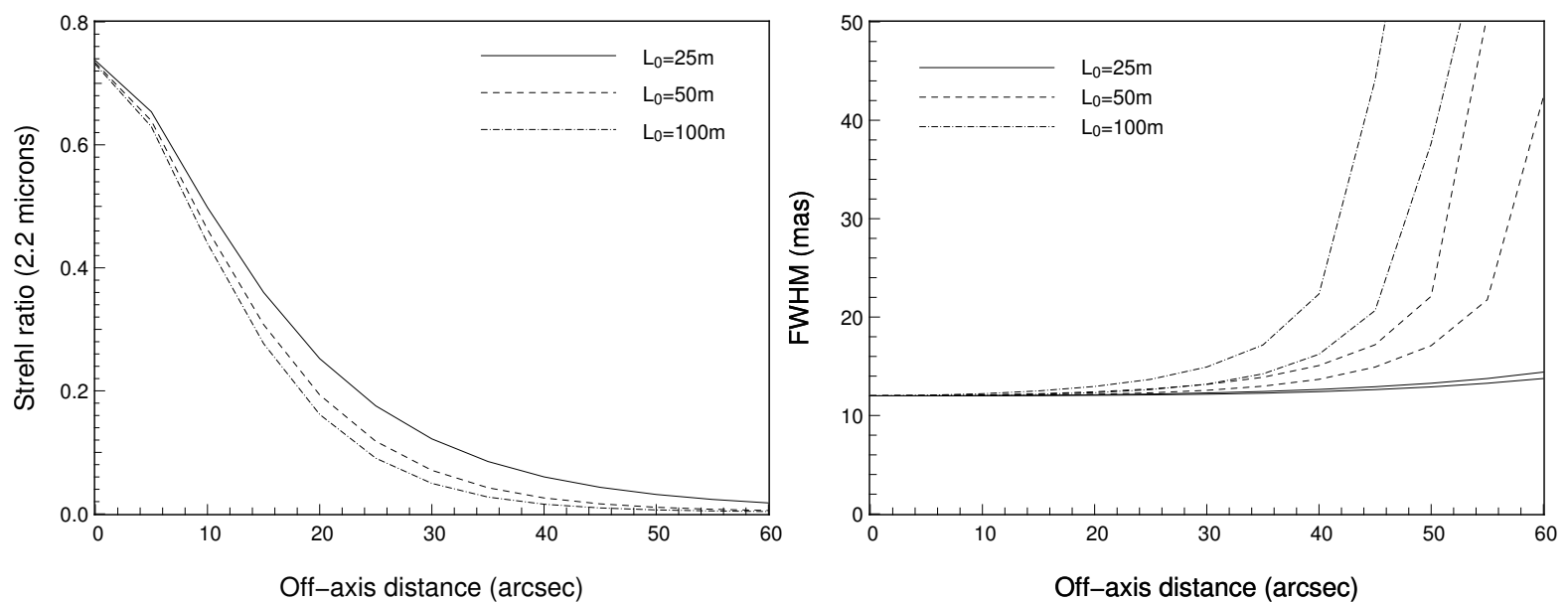

(b) Median seeing case
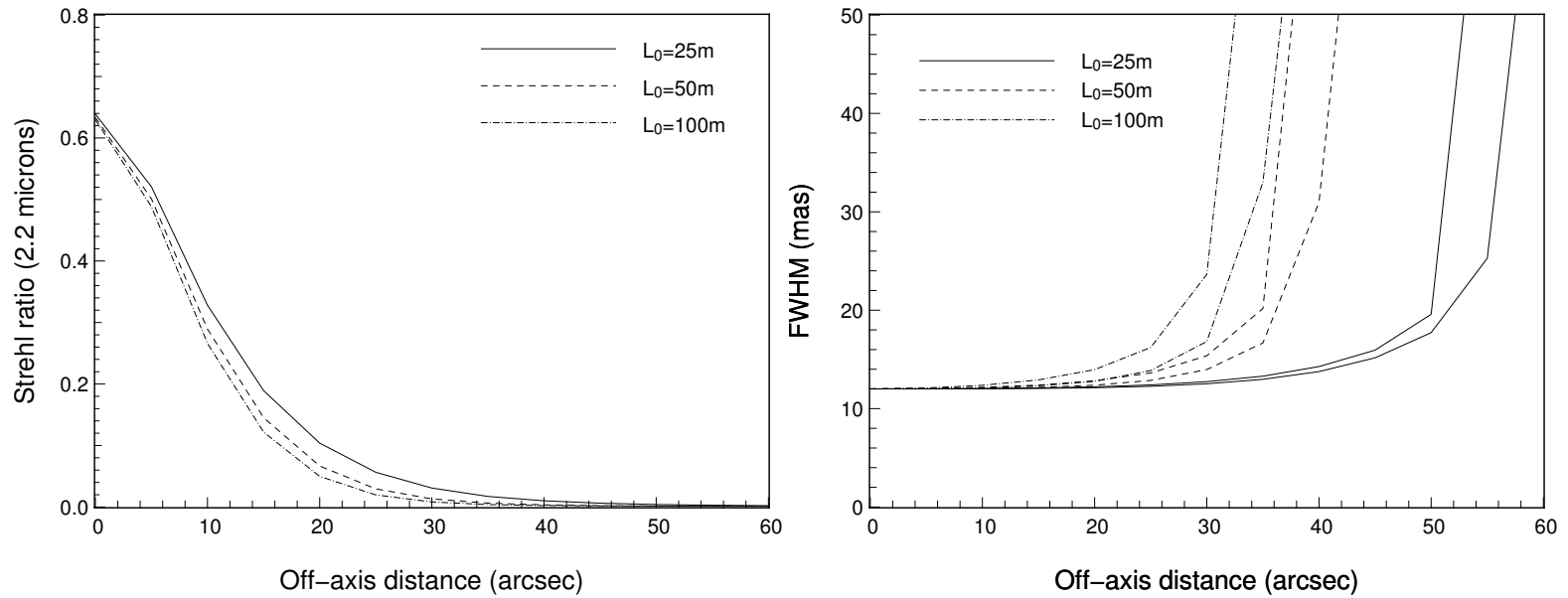

(c) Bad seeing case

Fig. 2. $K$-band Strehl ratio (left) and FWHM in mas (right) with respect to the $m_{\mathrm{V}}=12$ reference source off-axis distance (in arcsec), obtained with the Fourier-based simulation in the good (top), median (middle), and bad (bottom) case turbulence conditions and for outer scale values of $25 \mathrm{~m}$ (solid lines), $50 \mathrm{~m}$ (dashed lines), and $100 \mathrm{~m}$ (dot-dashed lines). For each turbulence and outer scale case, FWHM is plotted for the radial and transversal cuts of the PSF.

was assumed to be observed in the $K$-band at $30^{\circ}$ zenith angle. Optimizations led to an AO loop sampling frequency of $400 \mathrm{~Hz}$ with a loop gain of 0.4 (integral controller with gain), a loop delay of $3 \mathrm{~ms}$. End-to-end Monte Carlo simulations of a single Shack-Hartmann sub-aperture under these conditions demonstrated a noise level of $56 \mathrm{~nm}$ rms with sub-apertures of
$50 \mathrm{~cm}$. We do not claim that these parameters are still optimum; however, we think they represent a reasonable basis for a "high flux" case study.

Figure 2 presents the simulation results in terms of $K$-band Strehl ratio and PSF full width at half maximum (FWHM) with respect to the reference source off-axis distance in the good, 


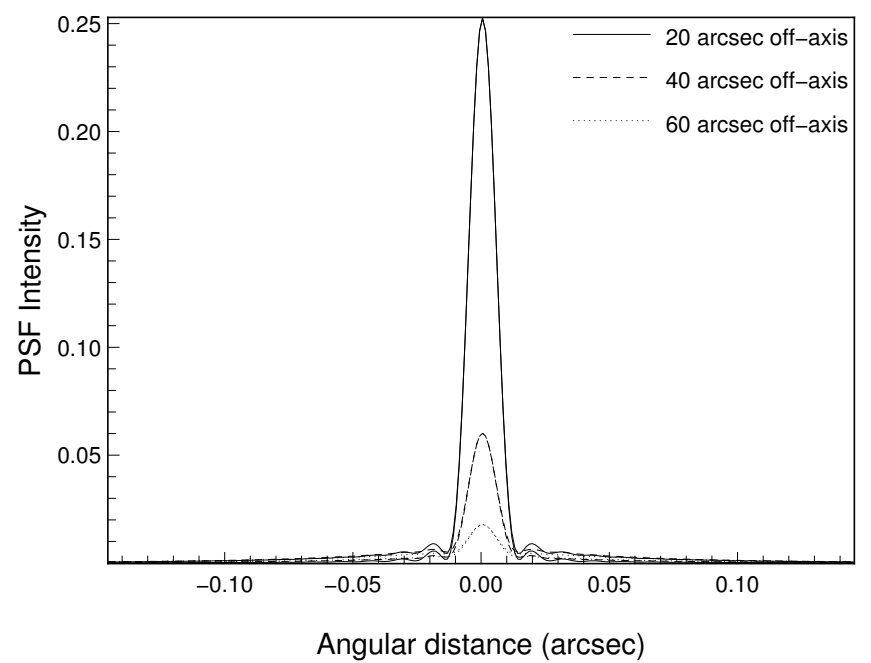

Fig. 3. Radial and transversal cuts of the E-ELT SCAO PSF obtained with the Fourier-based simulation under median seeing conditions with $L_{0}=25 \mathrm{~m}$. Reference source off-axis distances are 20" (solid lines), $40^{\prime \prime}$ (dashed lines), and 60" (dotted lines). The PSF intensity is normalized to the PSF $K$-band Strehl ratio: the maximum intensity is equal to the Strehl ratio $(25 \%, 6 \%$, and $\approx 2 \%$, respectively). Radial and transversal cuts appear superimposed.

median, and bad seeing cases and for three values of $L_{0}: 25,50$, and $100 \mathrm{~m}$. The influence of the seeing and outer scale on the Strehl ratio and more particularly on the anisoplanatism error are expected and have already been described in the literature:

- the better the seeing, the smaller the effect of the anisoplanatism on the Strehl ratio. For $L_{0}=25 \mathrm{~m}$ under good seeing conditions, the on-axis SR reaches $80 \%$, degrades to $74 \%$ under median seeing conditions, and to $64 \%$ under bad seeing conditions. Under good seeing conditions, the SR goes down to $\sim 8 \%$ at 60 arcsec off-axis distance while this level of correction $(\mathrm{SR} \sim 10 \%)$ is reached at $\sim 32$ arcsec off-axis under median seeing conditions and $\sim 20$ arcsec off-axis under bad seeing conditions.

- the larger the outer scale, the larger the effect of the anisoplanatism on the Strehl ratio (e.g. Marchetti \& Bonaccini 1998).

It can be seen in Fig. 2 that the FWHM of the SCAO PSF exhibits a large plateau at the diffraction limit, despite the increasing offaxis distance. This plateau extends up to a certain field angle, whose value depends on both the seeing and the outer scale. The better the seeing or the lower the outer scale, the larger this offaxis distance. It is remarkable that this diffraction-like pattern coexists with low Strehl ratios (below 0.20) and thus at angular distances much larger than the isoplanatic angle.

A hint for such behaviour of the E-ELT SCAO PSF is the study of Conan et al. (2003) that demonstrated a diffractionlimited turbulent PSF for a telescope diameter larger than $\sim 30-40 \mathrm{~m}$ and an outer scale up to $\sim 10 \mathrm{~m}$. We complement this study and show that at the E-ELT scale and under median seeing conditions, the off-axis SCAO PSF also preserves its coherent core at a distance that can be as large as $\sim 30$ arcsec and for an outer scale as large as $50 \mathrm{~m}$.

Figure 3 illustrates the evolution of the PSF profile with respect to the off-axis distance, under median seeing conditions for a relatively small outer scale value $(25 \mathrm{~m})$. It demonstrates the preservation of the PSF FWHM far from the reference source, despite the expected drop of SR. Hence, despite the low SR achieved at a 60 arcsec reference source off-axis distance, a

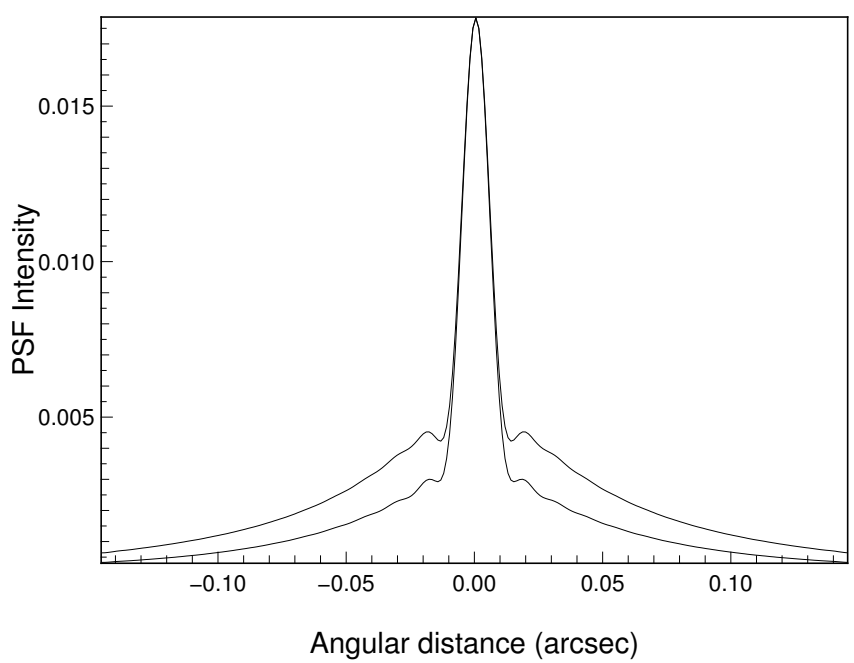

(a) $25 \mathrm{~m}$ outer scale case

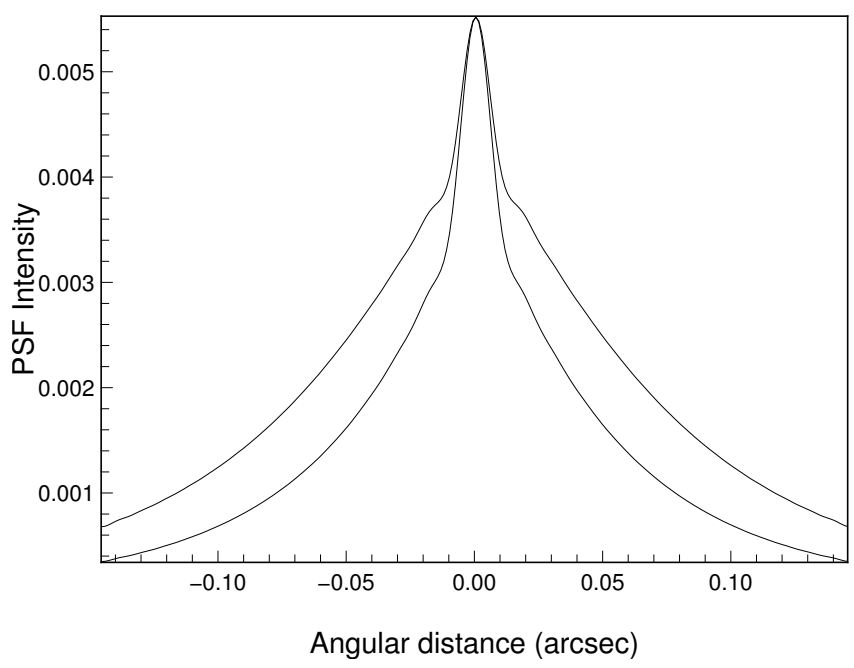

(b) $50 \mathrm{~m}$ outer scale case

Fig. 4. Radial and transversal cuts of the E-ELT SCAO PSF obtained with the Fourier-based simulation under median seeing conditions, $60^{\prime \prime}$ off-axis distance, and $L_{0}$ equal to $25 \mathrm{~m}$ (top) or $50 \mathrm{~m}$ (bottom). The PSF intensity is normalized to the PSF $K$-band Strehl ratio: the maximum intensity is equal to the $S$ trehl ratio $(\approx 2 \%$ and $0.6 \%$, respectively).

coherent core is still preserved when $L_{0}=25 \mathrm{~m}$ (i.e. less than the telescope diameter). This is better illustrated in Fig. 4a, which is a zoom of the PSF shown in Fig. 3. Figure 4b demonstrates the same behaviour for the larger outer scale value of $50 \mathrm{~m}$ : a coherent core is still present in spite of this very low SR and large outer scale value.

\section{Estimation of the anisoplanatism effect on the E-ELT SCAO PSF from end-to-end simulations}

\subsection{Description of the simulation software}

In order to counter-check the previous results, we have run endto-end simulations using our GPU-based end-to-end AO simulation platform COMPASS $^{1}$ (Gratadour et al. 2013).

\footnotetext{
1 https://projets-lesia.obspm.fr/projects/compass/ wiki/Wiki
} 
A description of this software package, together with its performance in terms of execution speed, as well as first E-ELTscale simulation results for MICADO, can be found in Clénet et al. (2013). In short, this software stack relies on the binding of a Yorick plugin built with the NVIDIA CUDA toolkit ${ }^{2}$ to provide GPU acceleration from within the Yorick environment. Inspired by $\mathrm{YAO}^{3}$, a CPU-based end-to-end adaptive optics simulation tool developed by F. Rigaut, it can be run through scripting and through GUI. It then allows an easy access to all parameters from within the Yorick session. Its main AO features are the following:

- on-line multiple layers Kolmogorov turbulence generation based the method described by Fried \& Clark (2008) extended to arbitrary outer scale values using Assémat et al. (2006), with various altitudes, strengths, speeds, directions and outer scales;

- multi-directional raytracing through turbulence;

- multiple natural or laser guide star wavefront sensors (either Shack-Hartmann, or pyramid, or roof sensor);

- centroiding using various algorithms: weighted centers of gravity $(\mathrm{CoG})$, thresholded $\mathrm{CoG}$, brightest pixels $\mathrm{CoG}$, or correlation;

- controlling using various algorithms: classical least square, CuReD (Rosensteiner 2011), minimum variance, linear quadratic Gaussian;

- multiple targets;

- multiple deformable mirrors.

\subsection{Simulation conditions and results}

For comparison with the Fourier-based results, we have run our end-to-end simulations with the same turbulence parameters as in Sect. 3.3, i.e. the ESO nine-layer turbulence profile, but only with the median seeing conditions. Similarly, the reference source was chosen as a $m_{\mathrm{V}}=12$ star with the MICADO transmission. The loop was run at $1000 \mathrm{~Hz}$, with a two frame delay and a gain of 0.3 . The WFS was a Shack-Hartmann with $50 \mathrm{~cm}$ sub-apertures, $4 \times 4$ pixels per sub-aperture, and a sub-aperture pixel size equal to the sub-aperture diffraction limit.

A first set of three simulations was performed with a fixed outer scale value, as in Sect. 3.3: $L_{0}=25 \mathrm{~m}$ at 30 arcsec offaxis distance, $L_{0}=25 \mathrm{~m}$ at 60 arcsec off-axis distance, and $L_{0}=50 \mathrm{~m}$ at 60 arcsec off-axis distance. A second set of simulations was performed using distinct $L_{0}$ values for each of the nine turbulence profile layers. The $L_{0}$ value of each layer was set to closely match the $L_{0}$ profile measured at Paranal Observatory by Dali Ali et al. (2010), as shown in Fig. 5.

As illustrated in Table 2, the first set of end-to-end simulations and the Fourier-based ones provide similar Strehl ratio values. The PSF images and profiles obtained for the different test cases, produced in Fig. 6, are closely comparable to those obtained with our Fourier-based code shown in Figs. 3 and 4. Most importantly, these end-to-end simulations, in particular those performed using the realistic outer scale profile (Fig. 7), confirm the Fourier-based simulation results in Sect. 3.3: despite the low SR, the off-axis E-ELT SCAO PSF is topped with an Airy-like pattern even far off-axis and for realistic and large outer scale values.

\footnotetext{
2 https://developer.nvidia.com/cuda-zone

3 http://frigaut.github.io/yao/index.html
}

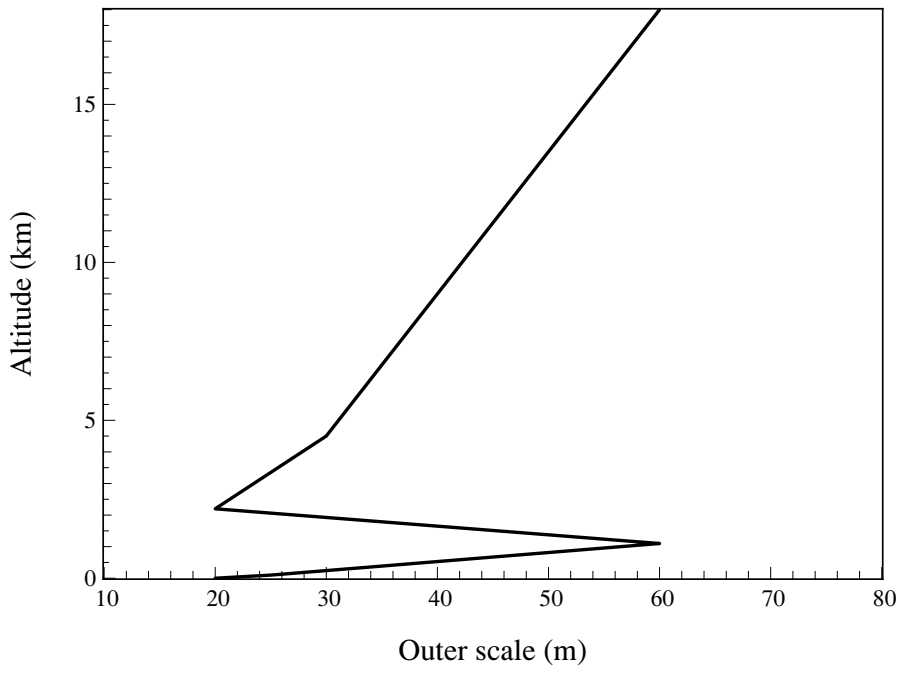

Fig. 5. Realistic outer scale profile set from Dali Ali et al. (2010) and used in the end-to-end simulation.

Table 2. $K$-band Strehl ratio for three different outer scale values and reference source off-axis distances obtained with the Fourier-based and the end-to-end simulation codes.

\begin{tabular}{cccc}
\hline \hline $\begin{array}{c}L_{0} \\
(\mathrm{~m})\end{array}$ & $\begin{array}{c}\text { Off-axis distance } \\
(\operatorname{arcsec})\end{array}$ & $\begin{array}{c}\text { SR Fourier } \\
(\%)\end{array}$ & $\begin{array}{c}\text { SR end-to-end } \\
(\%)\end{array}$ \\
\hline 25 & 30 & 12 & 13 \\
25 & 60 & 1.8 & 2.1 \\
50 & 60 & 0.58 & 0.66 \\
\hline
\end{tabular}

\section{Conclusion}

Using three different approaches, from purely analytical to endend, we have studied the effect of anisoplanatism on the E-ELT PSF obtained with a SCAO correction for different outer scale conditions. Beyond the expected degradation of the Strehl ratio with respect to the off-axis distance, the most important result is the Airy-like pattern visible at the top of the PSF profile even off-axis when the adaptive optics correction is very poor and for a large range of outer scale values.

If such a result could have been expected for our lowest simulated outer scale value $(25 \mathrm{~m})$, given the already diffractedlimited turbulent PSF demonstrated by Conan et al. (2003) for $L_{0}=10 \mathrm{~m}$, we show that the SCAO PSF coherent core is preserved for larger and realistic outer scale conditions at off-axis distance as large as $60^{\prime \prime}$. It results from the increase of the telescope diameter in conjunction with the effect of finite outer scale value, which reduces the contribution of low-order aberrations to the total wave-front residuals.

This study should be refined by adding two potentially important contributors to the low order aberration error budget: the vibration and the wind shake residuals. These contributors to low-order errors should degrade the Airy-like pattern by enlarging it and it would be necessary to check that this pattern is not suppressed.

If sufficiently preserved, this effect could provide the E-ELT instruments with some astrometric capabilities even in SCAO. It could also improve the accuracy of tip-tilt measurements made on off-axis natural guide stars used in LTAO or MCAO. 

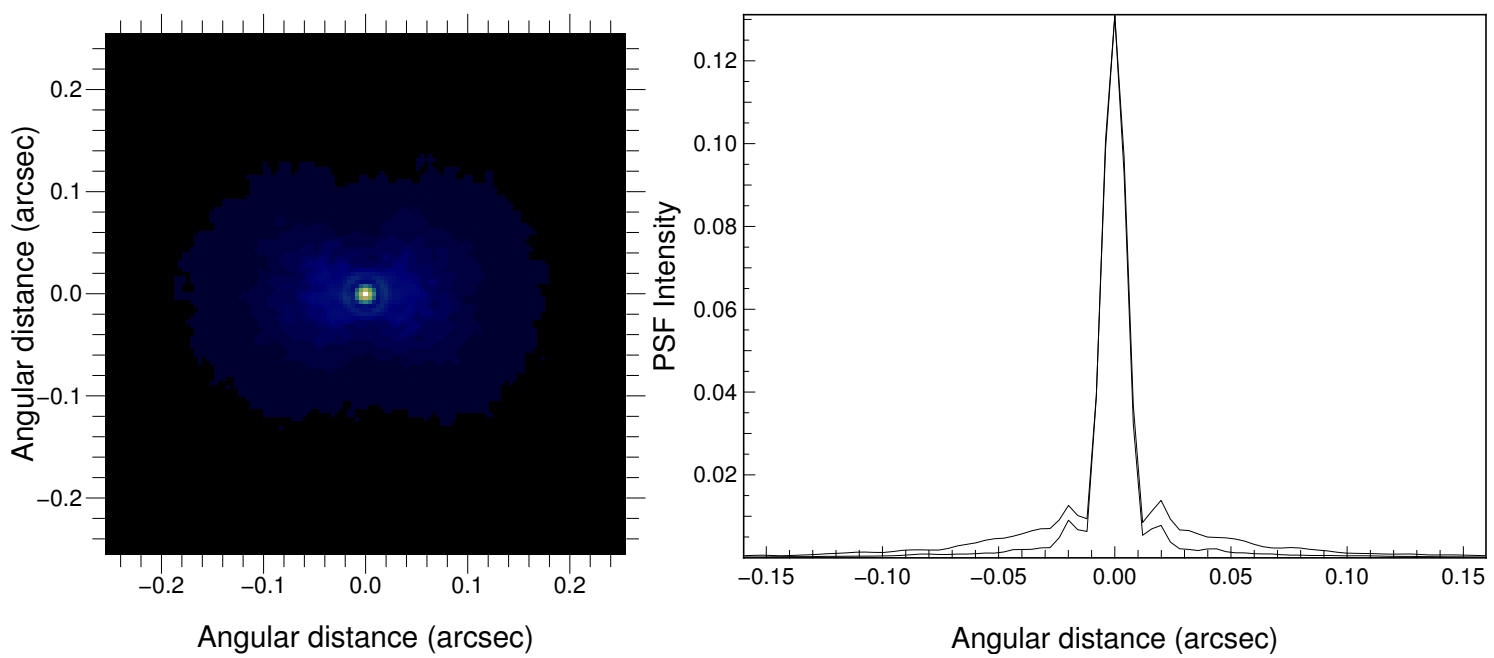

(a) $25 \mathrm{~m}$ outer scale and $30^{\prime \prime}$ off-axis distance case
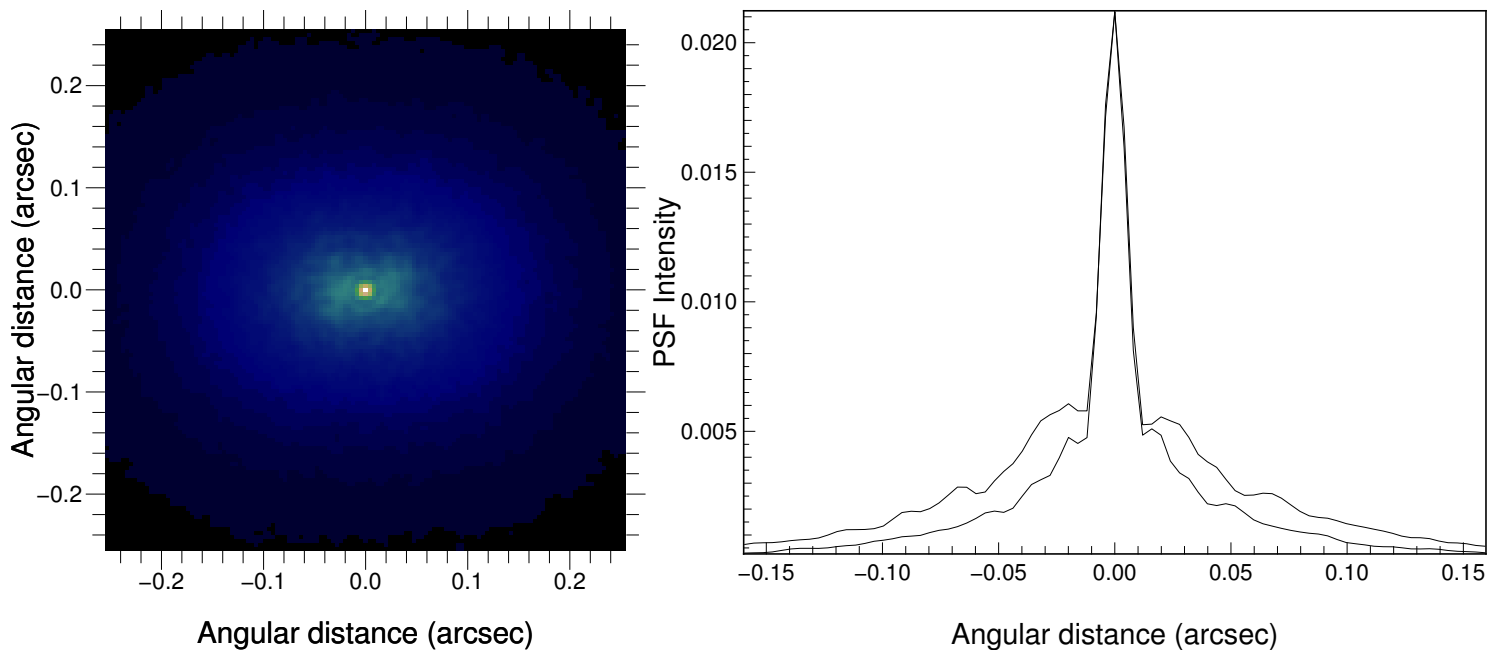

(b) $25 \mathrm{~m}$ outer scale and $60^{\prime \prime}$ off-axis distance case
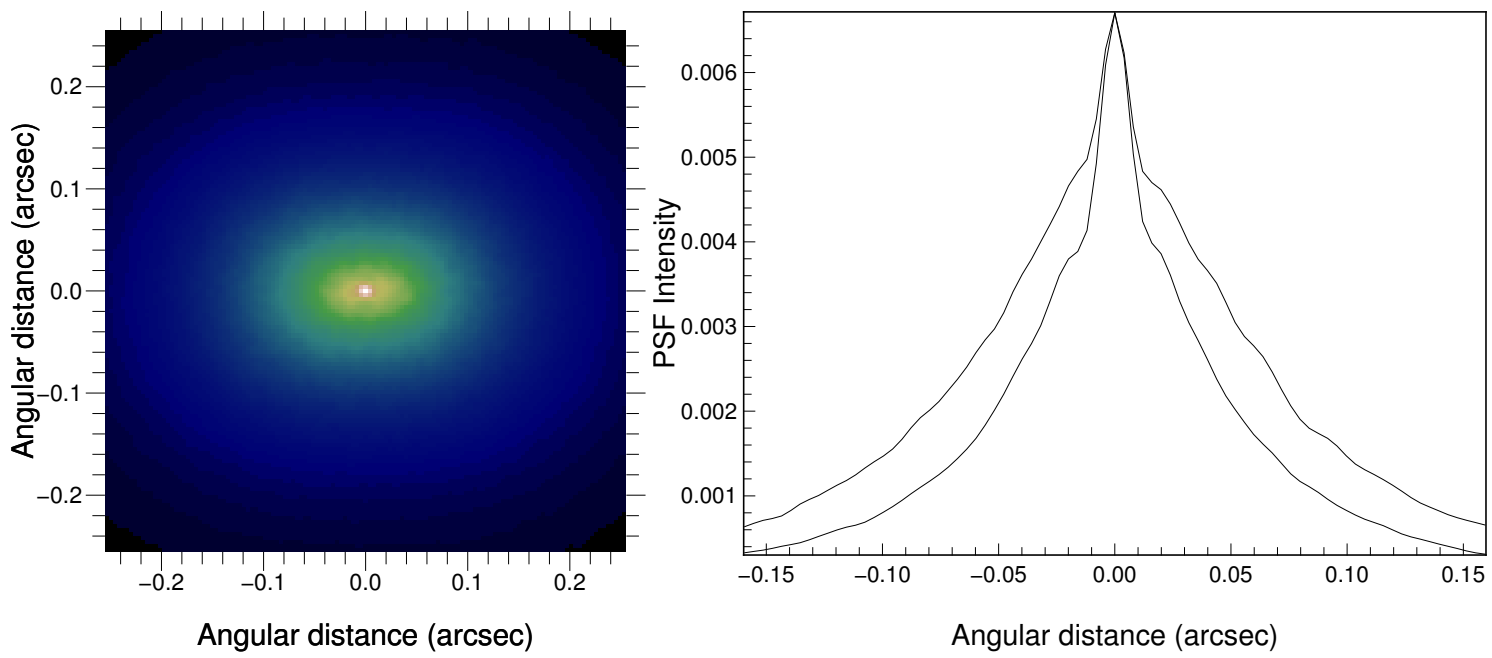

(c) $50 \mathrm{~m}$ outer scale and $60^{\prime \prime}$ off-axis distance case

Fig. 6. SCAO PSF images (left) and (radial and transversal) cuts (right) obtained in three different end-to-end simulation test cases. From top to bottom: a) $L_{0}=25 \mathrm{~m}$ and $30^{\prime \prime}$ off-axis distance; b) $L_{0}=25 \mathrm{~m}$ and $60^{\prime \prime}$ off-axis distance; and c) $L_{0}=50 \mathrm{~m}$ and $60^{\prime \prime}$ off-axis distance. For the PSF images, axis coordinates are the angular distance from the PSF maximum in arcseconds. For the PSF cuts, the abscissa coordinates are the angular distance from the PSF maximum in arcseconds, and the ordinate coordinates are normalized to the PSF Strehl ratio: the maximum intensity is equal to the Strehl ratio. 
Y. Clénet et al.: Anisoplanatism effect on the E-ELT SCAO point spread function. A preserved coherent core across the field
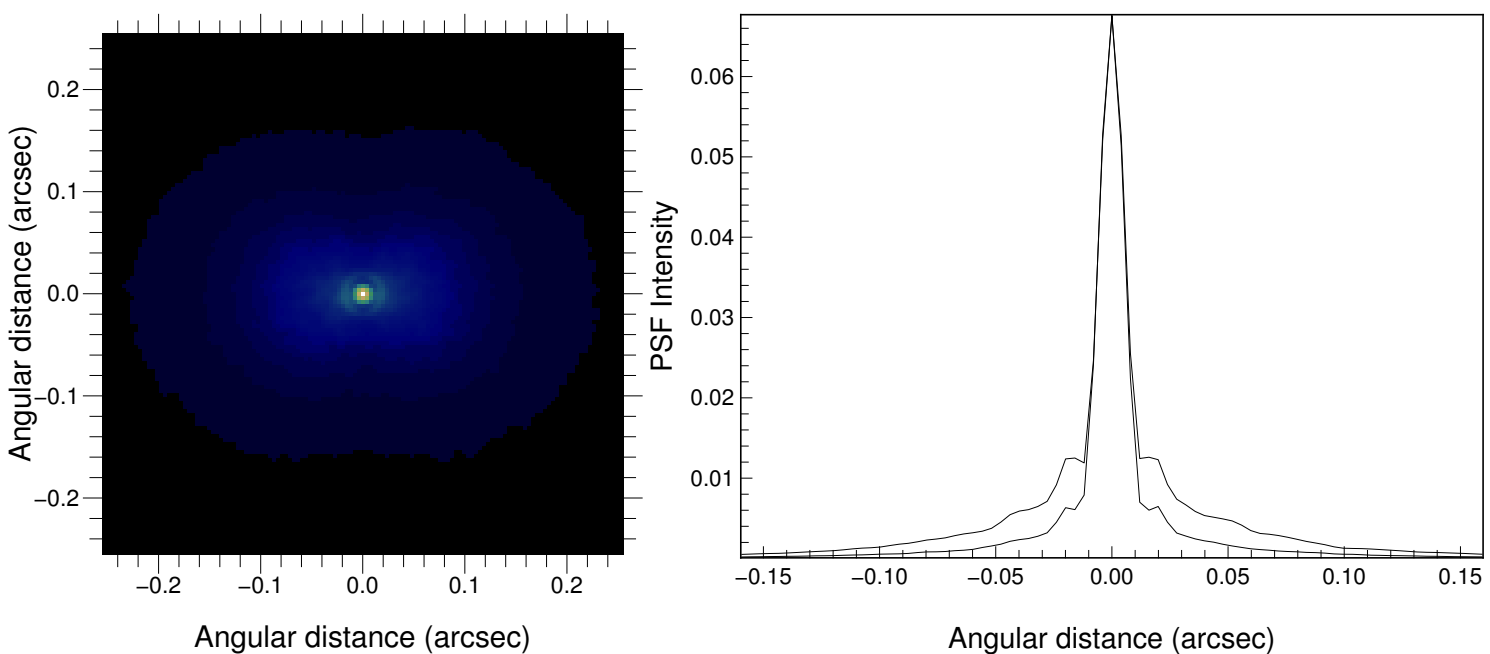

(a) Realistic outer scale profile and 30" off-axis distance case
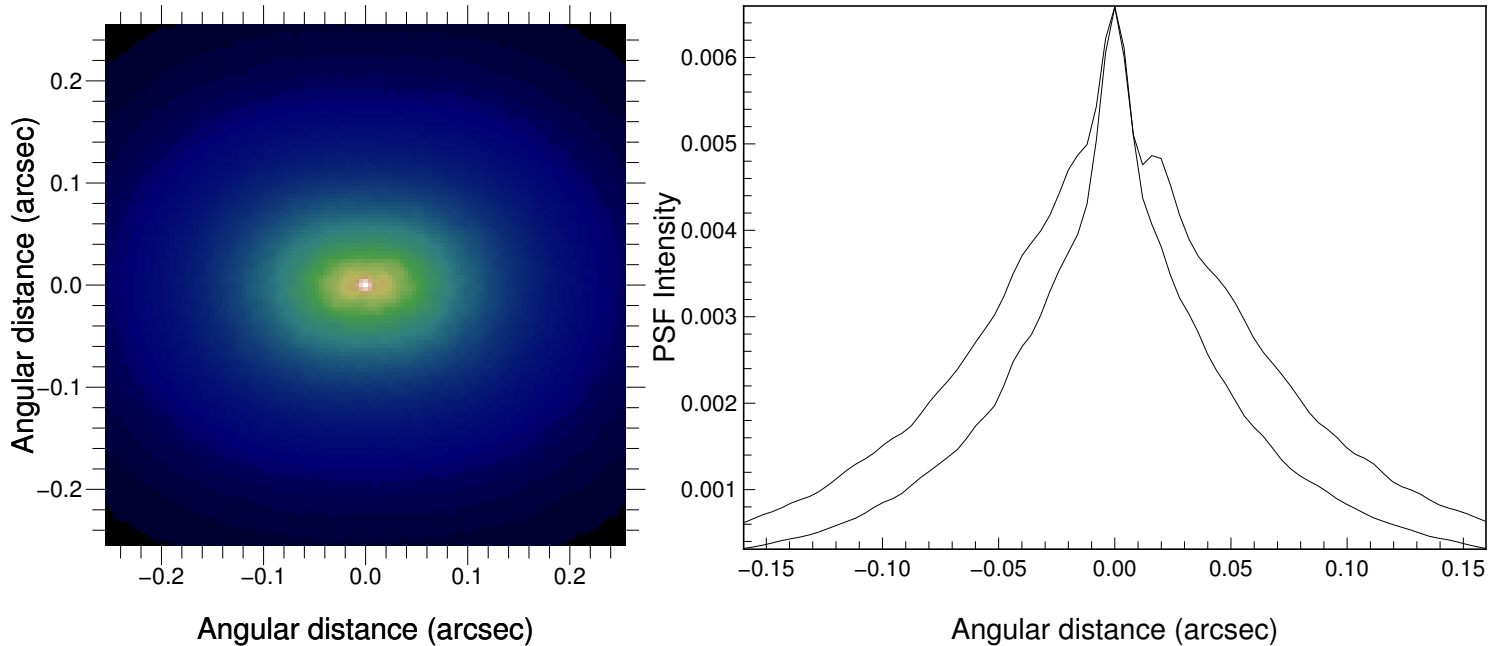

(b) Realistic outer scale profile and $60^{\prime \prime}$ off-axis distance case

Fig. 7. Same as Fig. 6 using the realistic $L_{0}$ profile of Fig. 5 with $30^{\prime \prime}$ off-axis distance a) and $60^{\prime \prime}$ off-axis distance b).

\section{Appendix A: Expression of the function $\sigma_{i j}$}

According to Chassat (1992), the function $\sigma_{j j}$ introduced in Eq. (4) is given by

$$
\begin{aligned}
\sigma_{j j}\left(2 \alpha h / D, 1, \pi D / L_{0}\right)= & 3.895(-1)^{n-m}(n+1) \\
& \times\left(K_{j j}^{1} H_{n+1,2 m, 0,14 / 3}\left(2 \alpha h / D, \pi D / L_{0}\right)\right. \\
& \left.+K_{j j}^{2} H_{n+1,0,0,14 / 3}\left(2 \alpha h / D, \pi D / L_{0}\right)\right)
\end{aligned}
$$

$K_{j j}^{1}$ and $K_{j j}^{2}$ are given in Table A.1.

Table A.1. $K_{j j}^{1}$ and $K_{j j}^{2}$ functions values.

\begin{tabular}{cccc}
\hline \hline & \multicolumn{3}{c}{$m \neq 0$} \\
& $m=0$ & even $j$ & odd $j$ \\
\hline$K_{j j}^{1}$ & 1 & $(-1)^{m}$ & $(-1)^{m+1}$ \\
$K_{j j}^{2}$ & 0 & 1 & 1 \\
\hline
\end{tabular}

$H_{n+1,2 m, 0,14 / 3}$ is given by

$$
\begin{aligned}
H_{n+1,2 m, 0,14 / 3}(\xi, \Lambda)= & \frac{1}{2 \sqrt{\pi} \Gamma(11 / 6)} \\
& \times \sum_{p=0}^{\infty} \sum_{q=0}^{\infty} \frac{(-1)^{p+q}}{p ! q !}\left(\left(\frac{\xi}{2}\right)^{2(q+m)} \Lambda^{2 p+2 q+2 n+2 m-5 / 3}\right. \\
& \times \Gamma_{1, n, m, p, q}+\left(\frac{\xi}{2}\right)^{2 p+2 q+14 / 3} \Lambda^{2 q} \\
& \left.\times \Gamma_{2, n, m, p, q}+\left(\frac{\xi}{2}\right)^{2 p+2 m} \Lambda^{2 q} \times \Gamma_{3, n, m, p, q}\right)
\end{aligned}
$$

with

$$
\begin{aligned}
& \Gamma_{1, n, m, p, q}= \\
& \frac{\Gamma(p+n+3 / 2) \times \Gamma(-p-q-n-m+5 / 6) \times \Gamma(p+q+m+1+n)}{\Gamma(q+2 m+1) \times \Gamma(p+2 n+3) \times \Gamma(p+n+2)} \\
& \Gamma_{2, n, m, p, q}= \\
& \frac{\Gamma(-p-q+m-7 / 3) \times \Gamma(p+n+3 / 2) \times \Gamma(q+11 / 6)}{\Gamma(p+q+m+10 / 3) \times \Gamma(-p+n+3 / 2) \times \Gamma(-p+1 / 2)}
\end{aligned}
$$




$$
\begin{aligned}
& \Gamma_{3, n, m, p, q}= \\
& \frac{\Gamma(p-q+n+m-5 / 6) \times \Gamma(-p+q-m+7 / 3) \times \Gamma(q+11 / 6)}{\Gamma(p+2 m+1) \times \Gamma(-p+q+n-m+23 / 6) \times \Gamma(-p+q-m+17 / 6)}
\end{aligned}
$$

where $\Gamma$ is the Euler gamma function.

\section{References}

Assémat, F., Wilson, R., \& Gendron, E. 2006, Opt. Exp., 14, 988 Chassat, F. 1992, Ph.D. Thesis, Université Paris Sud, France

Clénet, Y., Bernardi, P., Chapron, F., et al. 2010, Proc. SPIE, 7736, 77363

Clénet, Y., Gratadour, D., Gendron, E., et al. 2013, Proc. of the third Adaptive Optics for Extremely Large Telescope conference, eds. S. Esposito, \& L. Fini, online at http: //ao4elt3.sciencesconf.org/, 29

Conan, J.-M. 1994, Ph.D. Thesis, Université Paris XI Orsay, France

Conan, R., Avila, R., Sánchez, L. J., et al. 2003, Rev. Mex. Astron. Astrofís., 19, 31

Correia, C., Véran, J.-P., Herriot, G., et al. 2013, J. Opt. Soc. Amer., 30, 604 Dali Ali, W., Ziad, A., Berdja, A., et al. 2010, A\&A, 524, A73

Davies, R., Ageorges, N., Barl, L., et al. 2010, Proc. SPIE, 7734, 77340

Ellerbroek B. 1997, Appl. Opt., 36, 9456
Jochum L. 2008, Technical specifications for the conceptual design, prototyping, preliminary design of the M4 adaptive unit for the E-ELT, E-SPE-ESO-1060037

Fried D. 1982, J. Opt. Soc. Amer., 72, 52

Fried, D. L., \& Clark T. 2008, J. Opt. Soc. Amer. A, 25, 463

Fusco, T., Conan, J.-M., Mugnier, L. et al. 2000, A\&AS, 142, 149

Gendron, E., Abdelfattah, A., Gratadour, D., et al. 2014, Proc. SPIE, 9148

Gratadour, D., Sevin, A., Perret, D., et al. 2013, Proc. of the third Adaptive Optics for Extremely Large Telescope conference, eds. S. Esposito \& L. Fini, online at http://ao4elt3.sciencesconf.org/, 75

Jolissaint, L., Véran, J.-P., \& Conan, R. 2006, J. Opt. Soc. Amer. A, 23, 382

Marchetti, E., \& Bonaccini, D. 1998, Proc. SPIE, 3353, 1100

Muller, N., Michau, V., Robert, C., et al. 2011, Opt. Lett., 36, 4071

Neichel, B., Fusco, T., Conan, J.-M., et al. 2008, Proc. SPIE, 7015, 701573

Oberti, S., \& Kolb, J. 2008, E-ELT AO design inputs: relevant atmospheric parameters, E-SPE-ESO-276-0206 document

Racine, R., \& Ellerbroek, B. 1995, Proc. SPIE, 2534, 248

Ramsay, S., Casali, M., González, J. C. et al. 2014, Proc. SPIE, 9147, $91471 Z$

Rigaut, F., Véran, J.-P., \& Lai, O. 1998, Proc. SPIE, 3353, 1038

Rosensteiner, M. 2011, J. Opt. Soc. Amer. A, 28, 2132

Troxel, S., Welsh, B., \& Roggemann, M. 1994, Proc. SPIE, 2201, 137

Whiteley, M., Welsh, B., \& Roggemann, M. 1998, J. Opt. Soc. Amer. A, 15, 2097 